\title{
Sampling period determination for heart rate logging under an exercise regimen
}

\author{
M. MCCARTHY ${ }^{1} \&$ J. V. RINGWOOD ${ }^{2}$ \\ ${ }^{1}$ Eircom, Dublin and ${ }^{2}$ Department of Electronic Engineering, NUI Maynooth, Maynooth, Ireland
}

(Accepted 4 November 2005)

\begin{abstract}
Using a mathematical procedure, we determine appropriate sampling rates for logging heart rate, at a variety of exercise intensities. The mathematical procedure involves correlating exercise and heart rate data to determine a dynamical mathematical model, from which the frequency response of the relationship between exercise intensity and heart rate can be determined. The sampling rate is then straightforwardly deduced by making appropriate measurements on the frequency response curve. We show how careful consideration needs to be given to the choice of dynamical model structure and the work regimen, so that consistent and convincing conclusions can be drawn. We demonstrate that the dynamics of the workrate/heart-rate system are dependent on the nominal work/heart rate, but a 5-s sampling period, as used in many commercial heart rate monitors, appears to be adequate, especially when some averaging is performed before logging.
\end{abstract}

Keywords: Sampling rate, heart rate, system identification, frequency response

\section{Introduction}

Heart rate monitors with a recording facility are widely available, with a common minimum sampling interval of 5-s (Laukkanen \& Virtanen, 1998; Polar, 2003). Such monitors are of considerable use to athletes in guiding their training, principally to ensure that the exercise intensity is appropriate to the specific energy system being trained, as well as in pacing for training and racing.

In this paper we attempt to determine a valid heart rate sampling period for dynamical heart rate data logging, given the paucity of literature justifying the current adoption of a 5-s minimum interval. While many studies have examined the steady-state accuracy of heart rate monitors (Carroll, Godesn, \& Stone, 1991; Laukkanen \& Virtanen, 1998; MacFarlane, Fogarty, \& Hopkins, 1989; Polar, 2003), few researchers have examined the dynamical response of heart rate to changes in exercise intensity, which might lead to the specification of a suitable sampling period. One might speculate that this question has been given some consideration by developers of commercial heart rate monitors but, where they exist, such studies are not available in the public domain. Exceptionally, some heart rate monitors can $\log$ at a shorter interval than 5-s (e.g. the Polar ${ }^{\mathrm{TM}}$ Vantage), but generally memory limitations preclude the use of this interval, except for very short exercise bouts.

One of the applications of dynamical heart-rate logging is the determination of "anaerobic threshold" in athletes. Indeed, this is the motivation (Ringwood, 1999) that prompted the research reported in this paper, and has also been considered in such a context by others. However, perhaps critically, none have studied data from the workrate/heart-rate system at all exercise intensities. For example, Yamamoto et al. (1988) have looked at using a pseudo random binary sequence (PRBS)-like excitation signal for work rate but have not approached "anaerobic threshold" at all during the exercise protocol. Wigertz (1970) and Bakker, Struikenkamp and De Vries (1980) used sinusoid excitation of the work-rate/heart-rate system to model the ventilation and heart rate response to exercise. However, neither study stresses the entire work-rate range, relying instead on a small portion of the potential exercise range and thus leading to an incomplete system description.

The important issues addressed by this paper are as follows:

- A time domain technique is used to determine a mathematical model for the relationship between exercise intensity and heart rate, from which 
reliable frequency response (and consequently sampling period) estimates may be obtained.

- Small-amplitude stimulii are used to assess the dynamics of the work intensity/heart rate relationship at a number of levels to assess if the dynamics are consistent at all exercise intensities.

- An assessment will be made as to the validity of the 5-s sampling period currently adopted by most commercial heart rate monitors.

\section{Sampling period determination from frequency response}

The classical method for determination of the minimum sampling period of a signal is provided by the Nyquist-Shannon Theorem (NST) (Nyquist, 1928; Shannon, 1949), which specifies the minimum (Nyquist) sampling frequency as:

$$
f_{o}(H z) \geq 2 B(H z)
$$

where $B$ is the bandwidth of the signal. The bandwidth of a signal specifies that point in frequency above which all spectral components lie below a defined amplitude. Equation (1) can also be written in terms of angular frequency, $\omega$ ( $\mathrm{rads} \cdot \mathrm{s}^{-1}$ ), where $\omega_{o}=2 \pi f_{o}$. In some cases, for example where a signal is irregularly sampled (Vaidyanathan, 2001), the condition in (1) can be relaxed a little and this may have some relevance in the case of heart rate, where measurements of heart rate are available at each heart beat (measuring the previous interval). However, in commercial heart rate monitors these samples are subsequently aggregated and stored on a fixed time interval (e.g. 5 s). Therefore, such latitude does not apply in this case.

For sampling a system, such as the system relating heart rate to exercise intensity, the bandwidth, $B$, is determined in relation to the system frequency response, as depicted in Figure 1. The key idea here is that the frequency response represents (conceptually, at least) what would happen if the system were excited with a signal, $u(t)$, containing (uniform quantities of) all frequency components, as suggested by $U(f)$. The output spectrum, $Y(f)$, would then show the spectral properties of the system under this maximal case. A sampling period determined for this output signal, $y(t)$, would then be appropriate for this system. The input and output signals, $u(t)$ and $y(t)$, represent exercise intensity and heart rate respectively.

To determine the frequency response of the system, two approaches are possible: (1) a signal with a flat spectrum (as shown in Figure 1) is input to the system; or (2) individual sinusoids are input to "build up" a picture of the frequency response.

Only two signals have the flat spectrum required in the first possibility above: an impulse and white noise. An impulse, having infinite amplitude and negligible width, is physically unrealizable, while white noise demands continuous instantaneous changes in input signal and would be unrealistic in terms of a required exercise intensity profile. Possibility (2) above essentially allows the frequency response to be built up from samples of the spectrum at the individual sinusoidal frequencies. However, this requires a large number $(>10)$ of individual sinusoidal tests, with athletes required to follow an exact sinusoidal profile,
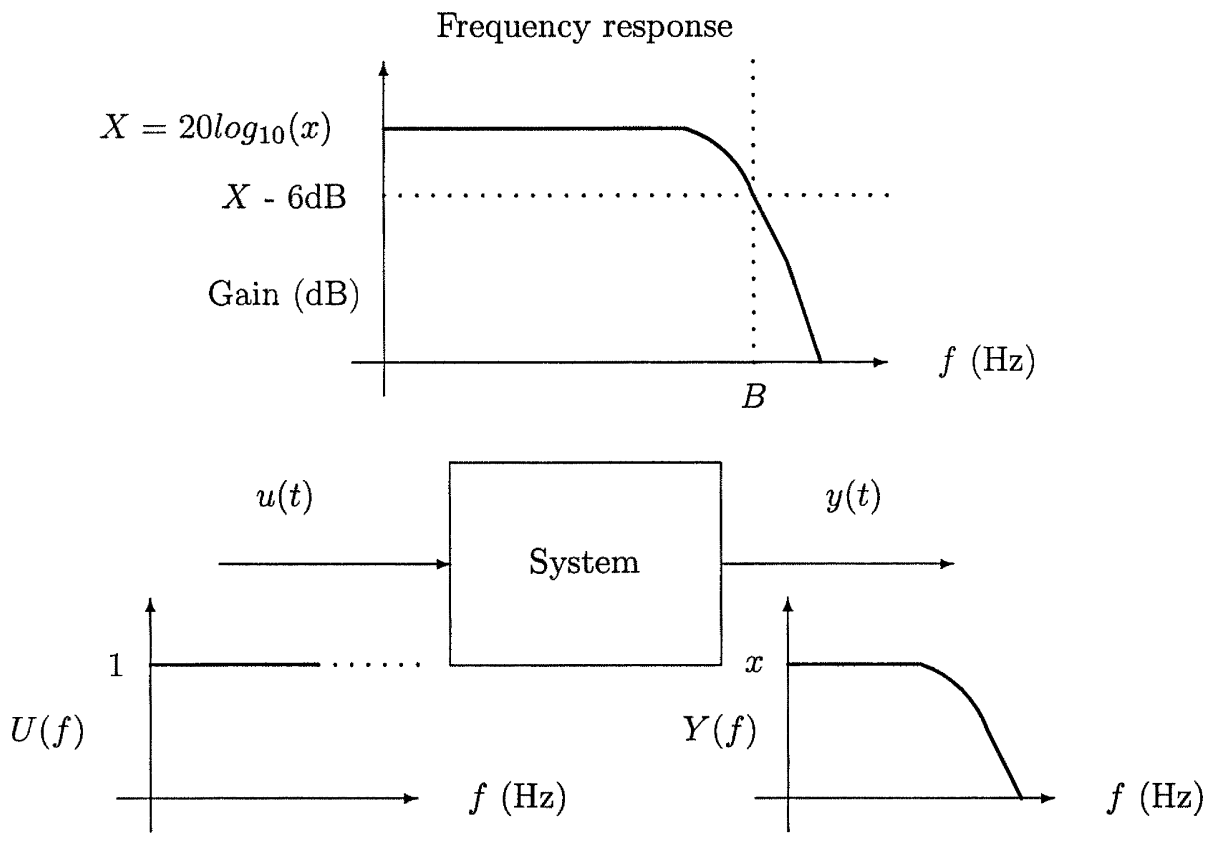

Figure 1. Bandwidth measures for signals and system. 
which is impractical, in terms both of difficulty and duration.

A useful signal, which has an approximately flat spectrum, is the pseudo random binary sequence (PRBS). This has recently been adopted by physiologists as optimal in providing maximum process information, while minimizing the duration of required experiments (Guild, Austin, Navakatikyan, Ringwood, \& Malpas, 2001), is well known as a suitable excitation for a variety of data-based modelling approaches (Ljung, 1999) and has been used before in heart rate excitation (Yamamoto et al., 1988).

In general, the frequency response of a system can be evaluated using an excitation with a non-flat spectrum, from:

$$
G(j \omega)=|G(j \omega)| \angle G(j \omega)=\frac{Y(j \omega)}{U(j \omega)}
$$

where $U(j \omega)$ and $Y(j \omega)$ represent the complex Fourier transforms of input and output signals respectively. A superior method, using similar measures, to evaluate the frequency response is provided by:

$$
\hat{G}(j \omega)=\frac{P x y}{P x x}
$$

where Pxy is the cross-spectral density of the system input and output and Pxx is the spectral density of the system input signal. An attempt was made to determine the frequency response (and hence a suitable sampling period) for a number of athletes using this method, but no cut-off frequency was apparent and the bandwidth is indeterminate (see McCarthy, 2005 for details).

This is due to the following issues:

- natural heart rate variability (Capurro, Diambra, \& Malta, 2003; Tulppo, Makikallio, Takala, Seppanen, \& Huikuri, 1996);

- measurement noise; and

- inaccuracies in interpolating heart rate and work intensity on to a regular sampling period.

All of the above issues are related to the time domain heart rate and work intensity signals. The direct spectral method of (3) makes no attempt to deal with these issues and significant distortion of the estimated frequency response occurs. The method outlined in the following section tries to deal with these issues directly.

\section{Frequency response estimation via time domain modelling}

In this section, a method for evaluating the frequency response by first creating a time domain, data-based model will be outlined, from which reliable bandwidth measures may be obtained.

\section{Time domain modelling}

The focus here is to produce a mathematical model that relates the heart rate time-series to the measured exercise intensity. In general, this model has been found to be non-linear (Ringwood, 1999), but to determine an analytical frequency response measure (in the following section), a linear formulation must be used. However, so as not to compromise the validity of a linear model, a number of linear models will be determined at various operating levels of heart rate/ exercise intensity. This has been shown to be a valid approach in data-based modelling (Takagi \& Sugeno, 1985) and has its origins in local linearization of non-linear systems about operating points (Athens, Dertouzos, Spann, \& Mason, 1974). In this research, a number of operating points will be examined, about which variations in exercise intensity will be induced. This provides an information-rich input signal, which will allow the determination of a mathematical system model from measurements of the system input and output signal. Such a practice, entitled system identification, is a well-established practice in dynamical modelling and control circles (Ljung, 1999).

In linear dynamical modelling, a set of discretetime data, sampled at regular intervals separated in time by the sampling period, $T$, is used to determine the parameters of a linear model of the form:

$$
\begin{aligned}
& y_{k}+a_{1} y_{k-1}+a_{2} y_{k-2}+\cdots+a_{n} y_{k-n}=b_{o} u_{k-d} \\
& \quad+b_{1} u_{k-d-1}+\cdots+b_{m} u_{k-d-m}+e_{k}
\end{aligned}
$$

where $m \leq n$, with $u$ and $y$ representing the input/ output data respectively. $e_{k}$ can represent an unmeasurable disturbance on the output, or measurement noise. Equation (4) can be written in the following form, which separates out the measurements from the parameters as:

$$
\begin{aligned}
& y_{k}=-a_{1} y_{k-1}-a_{2} y_{k-2}-\cdots-a_{n} y_{k-n}+b_{o} u_{k-d} \\
& +b_{1} u_{k-d-1}+\cdots+b_{m} u_{k-d-m}+e_{k}
\end{aligned}
$$

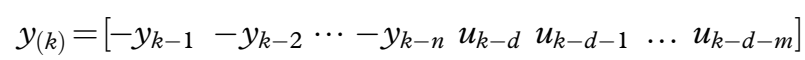

$$
\begin{aligned}
& \times\left[\begin{array}{c}
a_{1} \\
a_{2} \\
\vdots \\
a_{n} \\
b_{d} \\
b_{d+1} \\
\vdots \\
b_{d+m}
\end{array}\right]+e_{k}
\end{aligned}
$$




$$
=\phi \Theta+e_{k}
$$

Let's now suppose that an output/input data set $\left(y_{1}, \ldots, y_{N}\right) /\left(u_{1}, \ldots, u_{N}\right)$ is now available. Equation (7) can now be expanded to incorporate all these data as:

$$
Y_{k}=\Phi \Theta+E
$$

where

$$
\begin{aligned}
E & =\left[\begin{array}{c}
e_{1} \\
e_{2} \\
\cdots \\
e_{N-n}
\end{array}\right], Y=\left[\begin{array}{c}
y_{n+1} \\
y_{n+2} \\
\cdots \\
y_{N}
\end{array}\right], \\
\Phi & =\left[\begin{array}{cccccccc}
y_{n} & y_{n-1} & \cdots & y_{1} & u_{n-d+1} & u_{n-d} & \cdots & u_{n-d-m+1} \\
y_{n+1} & y_{n} & \cdots & y_{2} & u_{n-d+2} & u_{n-d+1} & \cdots & u_{n-d-m+2} \\
\vdots & \vdots & \cdots & \vdots & \vdots & \vdots & \cdots & \vdots \\
y_{N-1} & y_{N-2} & \cdots & y_{N-n} & u_{N-d} & u_{N-d-1} & \cdots & u_{N-d-m}
\end{array}\right]
\end{aligned}
$$

$E$ now represents the error in using the same parameter set with each set of data, termed the "equation error", as well as measurement noise and output disturbances. Equation (8) represents a set of linear equations that need to be solved for $\Theta$. A common solution to this set of overdetermined equations is provided by minimizing the sum of squares of errors $\left(E^{T} E\right)$ (Ljung \& Glad, 1994) as:

$$
\hat{\Theta}=\left(\Phi^{T} \Phi\right)^{-1} \Phi^{T} Y
$$

Several other methods are also available for system identification, using a variety of model forms, performance criteria, and optimization techniques (Ljung, 1999). That measurement noise and other disturbances, represented by $E$ in (8), are minimized is a major feature of the approach to frequency response determination adopted in this paper. This is in contrast to the method outlined in equation (2), where disturbances and noise are allowed to directly affect the spectral estimates.

One outstanding issue to be addressed is the determination of the model structure, via choice of the parameters $n, m$, and $d$ in (4). Usually, they are determined by calculating the performance (e.g. in a least squares sense) of various model structures on the input/output data. However, some care needs to be taken, since higher-order models will generally fit the data progressively better. Usually, this means that the model is starting to fit the noise in the data, as well as the underlying dynamics. This can be countered by examining, for the purpose of structure determination, the performance of each model on a data set different from that which determined the model parameters (sometimes termed a "validation" set). This second data set will have different additive noise, giving poorer model performance, if an attempt is made to fit to the noise in the original data. Another alternative is to use an "orderweighted" performance measure, such as Akakie's information theoretic criterion (AIC) or minimum description length (MDL) (Ljung, 1999), which provide a performance penalty commensurate with the complexity (e.g. number of parameters) of the model. A manual determination is also possible by looking at the incremental benefit of each increase in model complexity. Model parsimony is assumed to be achieved when further increases in complexity (order of numerator and denominator) only result in relatively minor improvements in model accuracy.

\section{Frequency response calculation}

Given that a parameter set (as in (10)) has been determined for the chosen linear model (as in (4)), it now remains to determine the frequency response of this model, from which the bandwidth (and, hence, the minimum heart rate sampling frequency) can be determined. Neglecting $e_{k}$ in (4), since we are focusing on the relationship between $y_{k}$ and $u_{k}$, and employing the $z$-transform, we get the discrete-time transfer function, $G(z)$, as:

$$
G(z)=\frac{Y(z)}{U(z)}=\frac{b_{0}+b_{1} z^{-1}+\cdots+b_{m} z^{-m}}{1+a_{1} z^{-1}+\cdots+a_{n} z^{-n}} z^{-d}
$$

where $z^{-1}$ represents the unit delay operator and $d$ is the number of steps of pure delay in the system. Given (11), the frequency response may now be easily evaluated as:

$$
G(\omega)=|G(\omega)| e^{j L G(\omega)}=\left.G(z)\right|_{z=e^{j \omega T}}
$$

where $\omega$ is frequency in $\mathrm{rad} \cdot \mathrm{s}^{-1}$. In general, as expressed in (12), $G(\omega)$ is a complex quantity, exhibiting both magnitude and phase properties. If the system in (11) is in factored form, then:

$$
G(\omega)=\frac{\left(e^{j \omega T}-z_{1}\right) *\left(d^{j \omega T}-z_{2}\right) \ldots \ldots}{\left(e^{j \omega T}-p_{1}\right) *\left(e^{j \omega T}-p_{2}\right) \ldots . .} e^{j \omega(-T d)}
$$

where $z_{i}$ and $p_{j}$ denote the zeros and poles of the model respectively. In terms of using frequency response to determine sampling period, we are primarily interested in magnitude, which can be easily evaluated, for different values of $\omega$, as:

$$
G(\omega)=\left.\frac{\left|b_{0}+b_{1} z^{-1}+\cdots+b_{m} z^{-m}\right|}{\left|1+a_{1} z^{-1}+\cdots+a_{n} z^{-n}\right|}\right|_{z=e^{j \omega T}}
$$




\section{Methods and experimental set-up}

A cycle ergometer was set up as shown in Figure 2. The ergometer enabled the participant to perform the exercise test while stationary, enabling easy connection of speed/work rate recording equipment to the computer. The ergometer primarily uses wind resistance to provide the load to simulate cycling forces (Murphy, 1999). Cycling induces less motion artefact into heart rate measurement than other alternative candidate exercises, such as running, since less upper body movement is involved. The following measurements were made:

- Heart rate, via a Polar ${ }^{\mathrm{TM}}$ chest strap and Printed Circuit Board Assembly (PCBA) receiver board connected to a laptop computer.

- Rear wheel speed, via a conventional wheel magnet and sensor, interfaced to the laptop computer.

To assist the participant in keeping pace, a conventional speed display was mounted on the handlebar. Heart rate and speed were logged on a laptop computer via a Personal Computer Memory Card International Association (PCMCIA) card connected to the heart rate and speed sensors. The (uncoded) Polar ${ }^{\mathrm{TM}}$ chest strap transmits a short burst of a $5-\mathrm{kHz}$ signal when an ECG QRS complex is electrically detected on the chest (Laukkanen \& Virtanen, 1998; Ruha, Sallinen, \& Nissila, 1997), which is subsequently received by the PCBA (Polar,

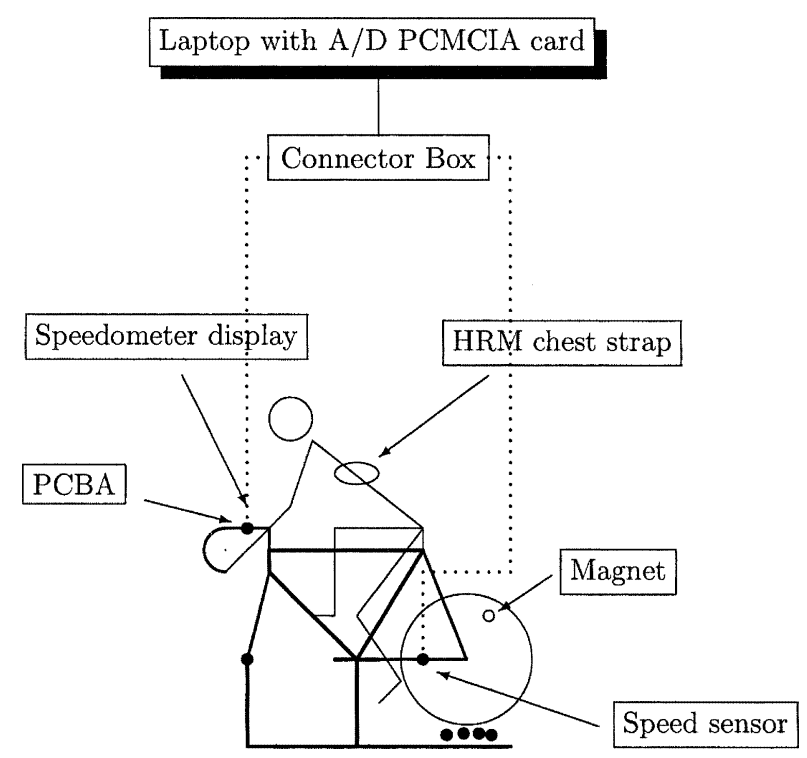

Figure 2. Experimental set-up. A/D, analog-to-digital conversion; PCMCIA, Personal Computer Memory Card International Association; HRM, heart rate monitor; PCBA, Printed Circuit Board "A" Assembly of Polar (1997).
1997) and a corresponding signal forwarded to the PCMCIA card in the computer. The accuracy of commercial heart rate monitors, using electrical detection of the QRS complex, is discussed in the literature (see, for example, MacFarlane et al., 1989). Speed (related to work intensity) is measured by detecting a wheel magnet passing a magnetically sensitive sensor, which passes on pulse-like signals to the PCMCIA card. A number of wheel magnets were used for improved speed resolution.

\section{Exercise protocol}

To examine the dynamical relationship between exercise intensity and heart rate at different operating points, an exercise protocol was chosen which applied individual stimuli at a small variety of exercise intensities. In addition, the exercise stimulus must have certain properties, if it is to be useful in eliciting the complete dynamics at any operating point:

- It must have broad frequency content, usually assured by having a variety of rapid changes in the signal (Ljung, 1999).

- It must be of sufficient amplitude to provide a measurable response in the heart rate signal. This not only relates to the amplitude of the exercise excitation, but also to the frequency content (rate of transition).

- It must be of sufficiently low amplitude that the deviation from the operating point does not violate the assumption of local linearity, allowing a linear model to be synthesized.

- It must be sufficiently long so that the averaging effect of the data-based modelling algorithms can be used to significantly reduce the effect of measurement noise and other disturbances.

- It must be sufficiently short so that it can be completed by the weakest individual.

- It must exercise, ideally, the complete range of heart rate.

The chosen exercise protocol is as shown in Figure 3 and is deemed to be the best compromise in fulfilling the objectives listed above. Note that a Tacx Eco Power air-trainer was used, which gives a resistance roughly proportional to speed cubed, similar to real cycling. Some equivalence of the relationship between speed and power (in watts) can be had at low speed (i.e. $15 \mathrm{~km} \cdot \mathrm{h}^{-1} \cong 11 \mathrm{~W}$ ).

\section{Participants}

To draw broad conclusions, healthy individuals of varying athletic ability were recruited, who also varied in size and age. From a poll of 10 full trials, 


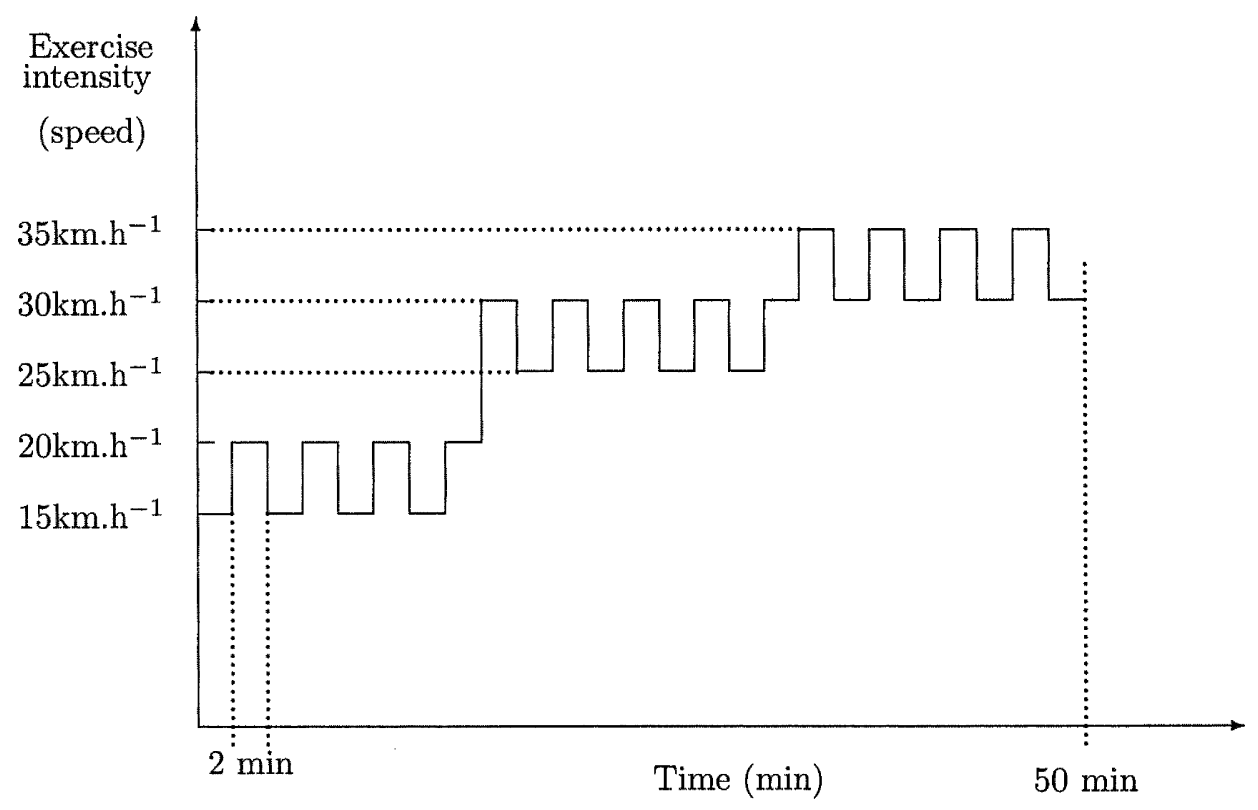

Figure 3. Exercise protocol.

five male participants (referred to as P1 to P5 in the remainder of the paper) were selected, eliminating:

- recordings that contained a large proportion of recording artefacts or logging malfunctions (recall that each participant must undergo three separate exercise sessions), and

- ECG traces containing significant irregularity (e.g. peak splitting), making calculation of interpeak period very difficult.

Of the five participants finally selected, one was sedentary, one was recreationally active, and three were competitive athletes in non-cycling-related aerobic sports. A medical questionnaire was administered to all participants. All five participants were competent cyclists. Table I outlines some of the participants' details.

\section{Data pre-processing}

Once the experimental data have been successfully recorded, the stored data must be pre-processed before modelling. The steps involved in this processing are as follows:

1. The heart rate and exercise intensity signals are digitized traces of the PCBA and speedometer signals respectively. The pulses in these traces, which represent heart beats (ECG QRS complexes) and wheel revolutions respectively, must be detected and converted into beat-by-beat heart rate and "per-wheel" revolution bicycle speed.
Table I. Participants' details.

\begin{tabular}{lccc}
\hline Participant & Height $(\mathrm{m})$ & Body mass $(\mathrm{kg})$ & Age \\
\hline P1 & 1.78 & 82 & 28 \\
P2 & 1.85 & 95 & 40 \\
P3 & 1.88 & 85 & 42 \\
P4 & 1.73 & 86 & 24 \\
P5 & 1.73 & 68 & 31 \\
\hline
\end{tabular}

2. Outliers in the recorded data sets (exercise intensity and heart rate) were removed using an adaptive threshold based on previous values in the data set and manual/visual inspection of exercise intensity and heart rate graphs.

3. In preparing the exercise intensity (input) and heart rate (output) data for modelling, the raw data sets for each participant were interpolated to give values at equally spaced points in time, since the models described using (11) require a consistent sampling period. This was performed by fitting cubic splines to the data (de Boor, 1978) and re-sampling at $1 \mathrm{~Hz}$.

4. The interpolated data were then segmented into low, medium, and high work rate sections, to allow model structures to be developed for participants at each principal intensity of work rate.

5. Data for each intensity of work rate for each participant were then de-trended (mean reduced to zero) and then subdivided into training and verification data, following standard system identification procedures (Ljung, 1999) to first train and then subsequently validate the models under development. 


\section{Results}

First, indicative results are shown that illustrate the operation of the analysis technique, from data logging to sampling period determination. Then, we provide an overview of the results, from which general conclusions can be drawn.

\section{Sample single participant results}

An exercise protocol similar to that in Figure 3 was followed, with the levels and timing of the speed adjustment communicated to the participant (P1) as the test progressed. The actual speed profile achieved and the resulting heart rate profile (following preprocessing) are both shown in Figure 4.

To perform a fair comparison of models, for different participants and exercise intensities, a consistent model structure was determined for all models as $n=2, m=1$, and $d=2$. This was arrived at by (a) calculating loss functions for all data segments (different participants and intensities) as:

$$
\mathcal{f}_{p, i}(n, m, d)=\sum_{k=1}^{K(p, i)}(y-\hat{y})^{2}
$$

where $p$ is the number of the participant, $i$ is the exercise intensity, and $K(p, i)$ is the number of data points available for each participant/intensity, with $y$ and $\hat{y}$ the actual and modelled heart rate. The $\mathcal{F}_{p, i}(m, n, d)$ may be combined (additively) to give a single, representative, loss function over all the data, as:

$$
\mathcal{f}(n, m, d)=\sum_{p=1}^{5} \sum_{i=1}^{3} \mathcal{F}_{p, i}(n, m, d)
$$

From this loss function, the structure was determined manually from experience, by looking for

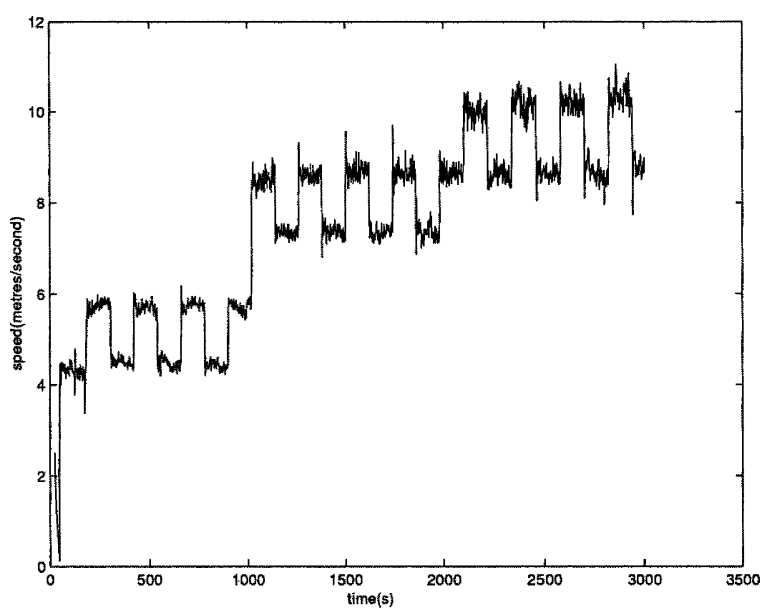

transitions in order that gave especially good drops in loss function, while penalizing higher orders (complexity). The loss function, for variations in $n$ and $m$, is shown in Figure 5. The greatest loss reduction for variation in $n$ occurs from $n=1$ to $n=2$, highlighting $n=2$ as an obvious candidate, while $m=2$ gives the lowest loss for $1 \leq m \leq 5$. Both selections give an acceptably low order (complexity).

As an example, the model determined for participant 1 at exercise intensity 1 (lowest) was:

$$
G(z)=\frac{0.646 z^{-2}}{1-0.729 z^{-1}-0.035 z^{-2}}
$$

For this case, the modelled and actual heart rate signals are compared in Figure 6 for the medium intensity (by way of example). The normalized (log magnitude) frequency response for the system in equation (17) is calculated according to the procedure outlined in the section entitled 'Frequency response calculation' and is plotted in Figure 7. The plot shows that, for this example, the frequency response drops by approximately $13 \mathrm{~dB}$ at $0.2 \mathrm{~Hz}$, indicating that the content of the heart rate signal is significantly reduced above $0.2 \mathrm{~Hz}$, indicating that a sampling period of $5 \mathrm{~s}$ is appropriate.

\section{Results overview}

Table II shows the gain reduction (from $0 \mathrm{~dB}$ ) for the various participants and exercise intensities at $0.2 \mathrm{~Hz}$ (corresponding to a sampling period of $5 \mathrm{~s}$ ).

A number of features are noteworthy. In particular, the standard deviation is large across different intensities for the same participant, indicating that there is a disparity in the dynamics at different intensities. However, in contrast, standard deviations are relatively small across different participants for comparable intensities, which indicates good consistency across participants. In general, there is a

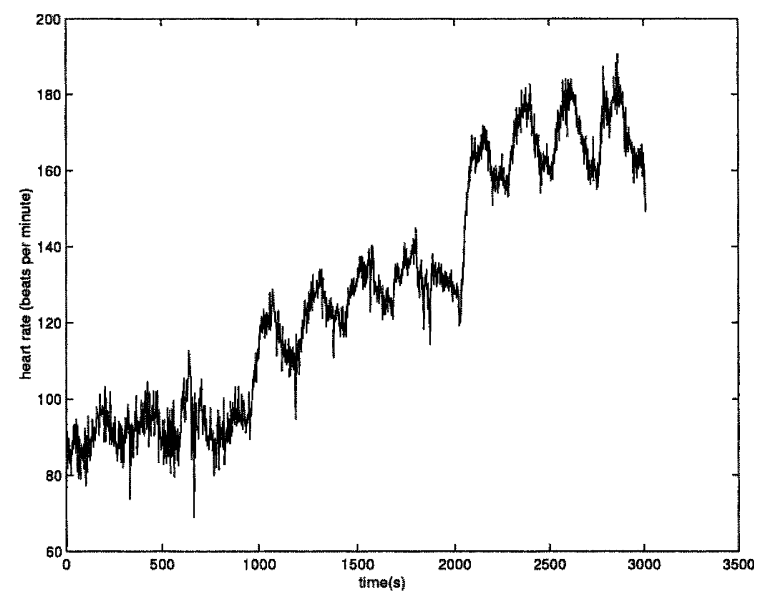

Figure 4. Typical processed speed and heart rate recordings. 


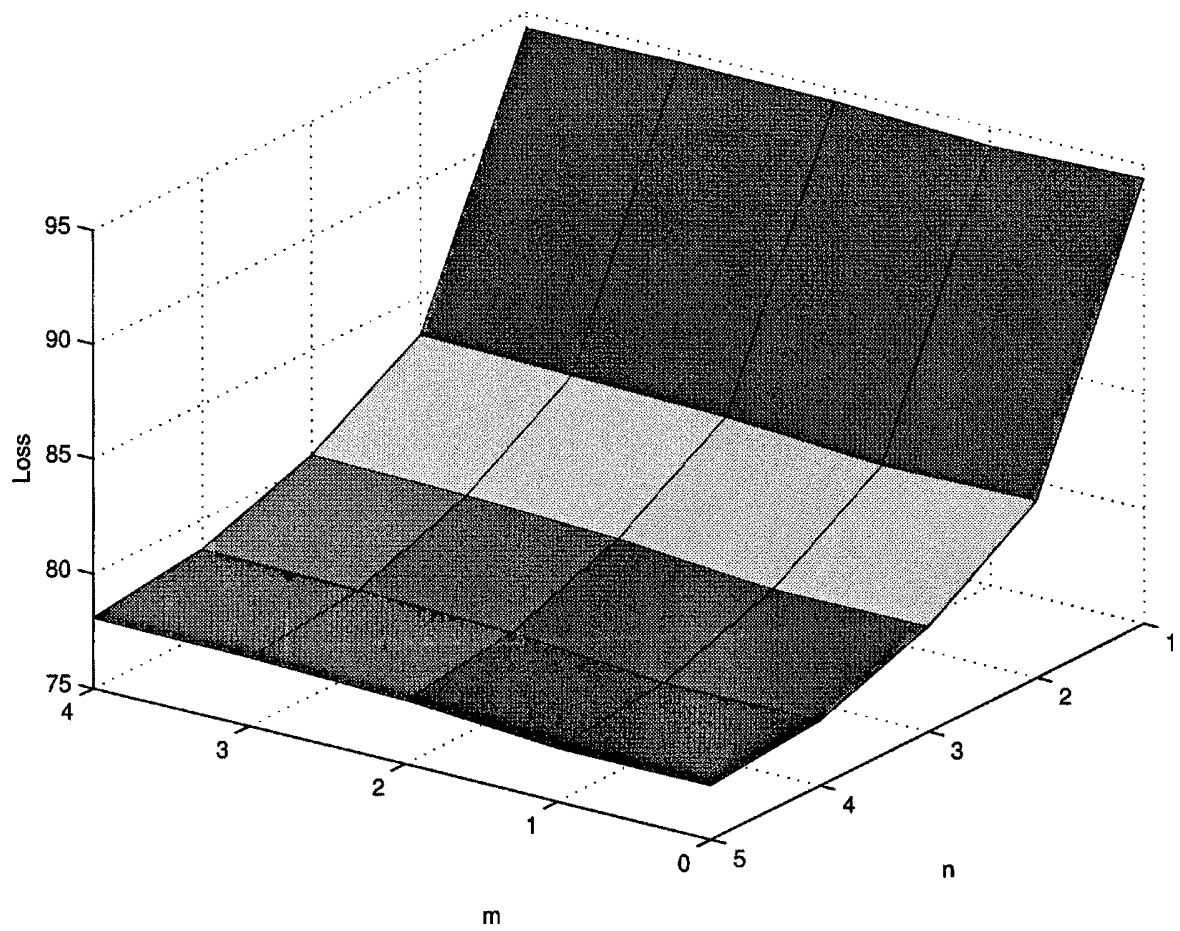

Figure 5. Loss function for model order determination.

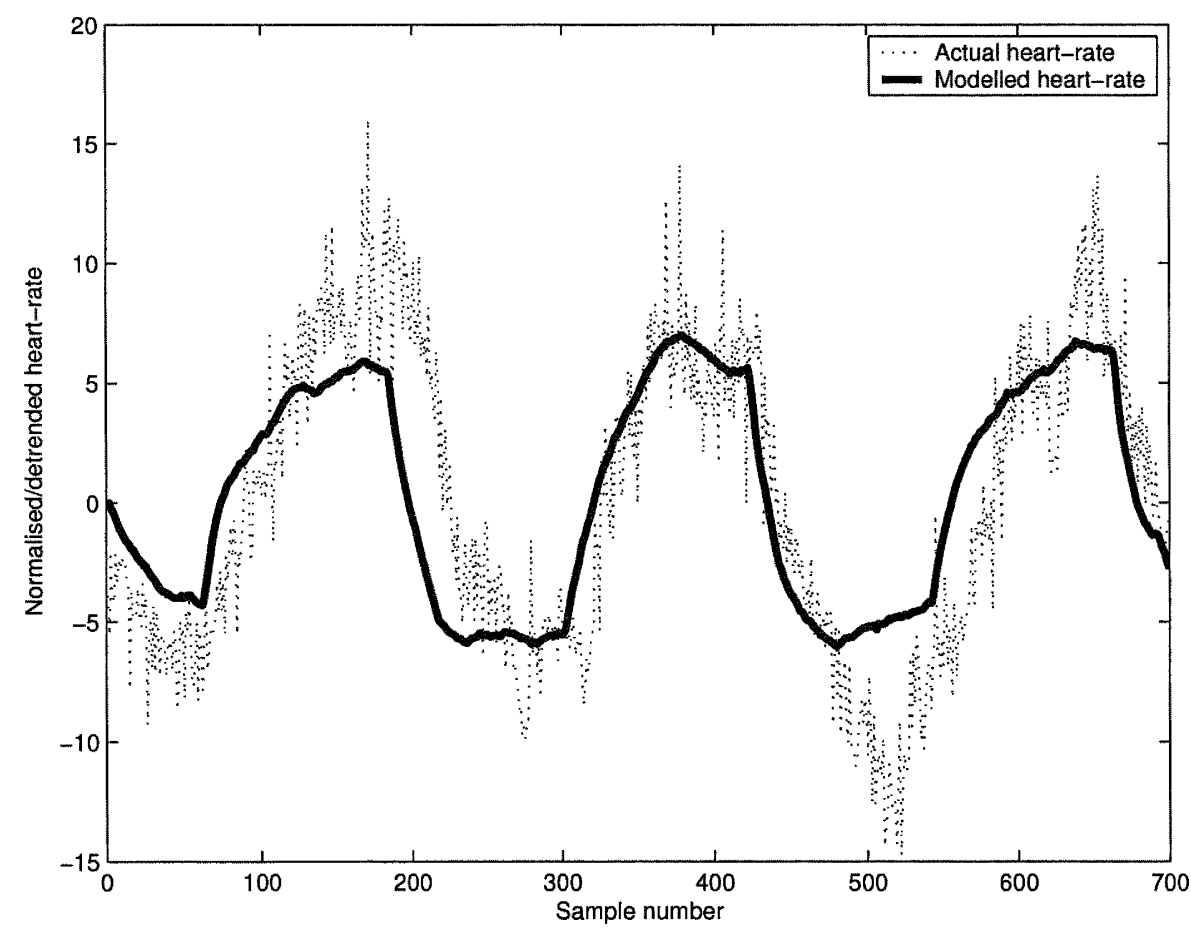

Figure 6. Modelled and actual heart rate comparison.

significant drop (between 12.5 and $22 \mathrm{~dB}$ ) in the magnitude response (from $0 \mathrm{~dB}$ ) at $0.2 \mathrm{~Hz}$ at all exercise intensities, but most significantly so for the medium (level 2) intensity. The magnitude response is slowest to drop at the lowest (level 1) intensity. Figure 8 shows the averaged frequency responses for all three exercise intensities and the standard deviations. There are clear differences in the dynamics between exercise intensity and heart rate at different exercise intensities/heart rates, with the fastest dynamics (highest bandwidth) at the lowest intensity. 


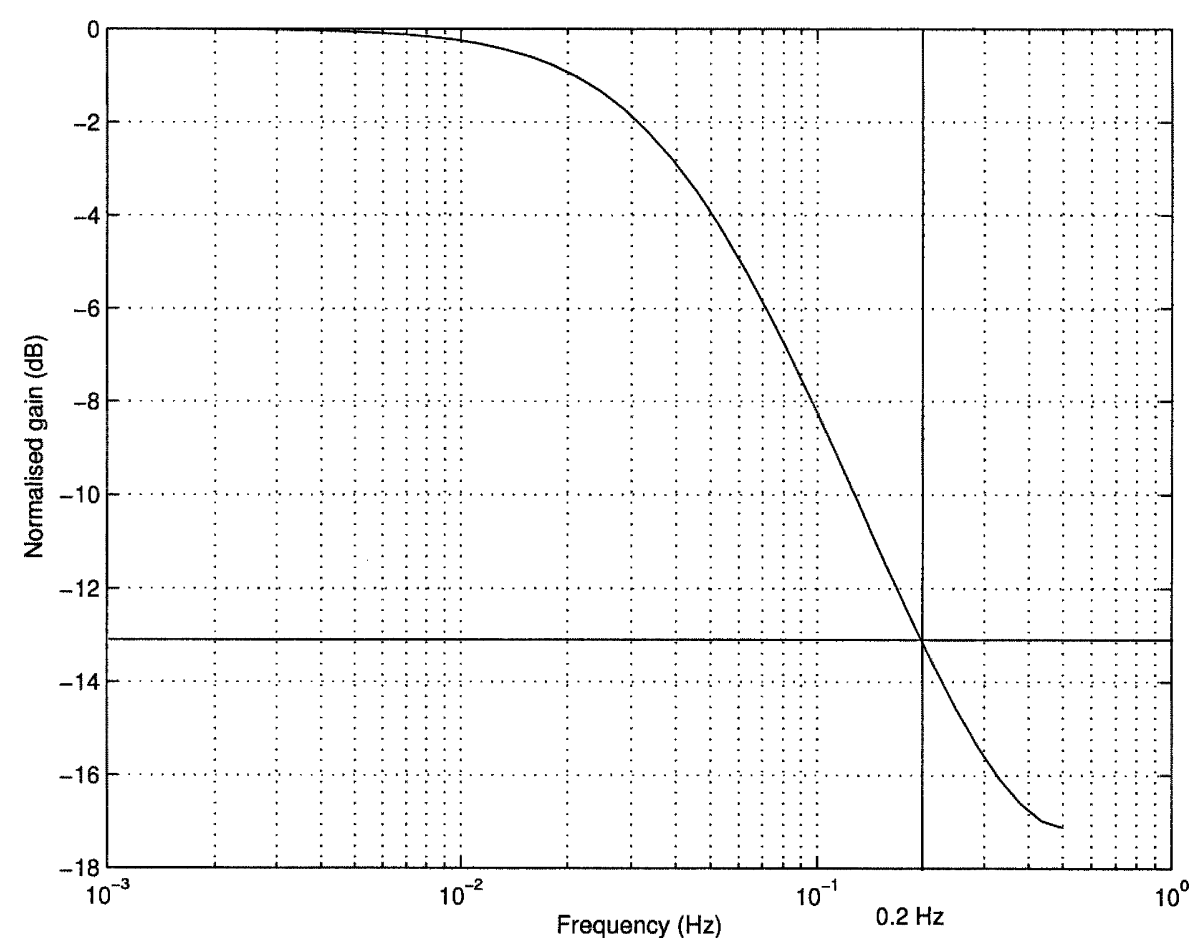

Figure 7. Frequency response for participant P1 at low intensity.

Table II. Decibel drop in frequency responses at $0.2 \mathrm{~Hz}$.

\begin{tabular}{lrrrrrrr}
\hline Exercise Intensity & P1 & P2 & P3 & P4 & P5 & Mean \\
\hline 1 & -12.56 & -9.25 & -10.22 & -10.54 & -17.53 & -11.84 \\
2 & -24.07 & -17.77 & -20.30 & -22.32 & -22.56 & -21.29 & -2.19 \\
3 & -15.71 & -19.51 & -23.28 & -14.41 & -13.47 & -17.28 \\
Mean & -17.44 & -15.51 & -17.94 & -15.76 & -17.82 & -15.88 & 4.07 \\
$S$ & 5.95 & 5.49 & 6.84 & 6.00 & 4.56 & 6.87 \\
\hline
\end{tabular}

\section{Conclusions}

The results clearly show that the speed of response in heart rate depends on the nominal intensity at which the exercise stimulus is applied. It appears that heart rate is most responsive when the exercise intensity is low, but the slowest rate of response is, in fact, at the "medium" intensity rather than the highest intensity. The fact that the dynamics are different at different exercise intensities has important connotations for the use of models [such as Hammerstein and Wiener, common in the physiology literature (Dempsey \& Westwick, 2004; Kawada et al., 2003)], which lump any non-linearity into a static characteristic, with the dynamics isolated in a single constant, linear transfer function. Clearly, such mathematical models will not provide an adequate representation of the variability in dynamics with operating point.

Recall that the frequency response between exercise stimulus and heart rate represents the maximum frequency content of the heart rate signal and can therefore be used to determine an appropriate sampling rate for the heart rate signal. In essence, the heart rate signal should display no significant content at frequencies above half the sampling period (i.e. $0.1 \mathrm{~Hz}$ ), if the signal is to be faithfully represented by its sampled version. This is clearly not the case here (from Figure 8), but there is considerable reduction in the spectrum after $0.01 \mathrm{~Hz}$. Even if the content is not zero at $0.1 \mathrm{~Hz}$, the signal can be band-limited to $0.1 \mathrm{~Hz}$ by appropriate low-pass filtering or averaging, which is a practice normally carried out in most commercial heart rate monitors - that is, a 5-s "average" is normally calculated (see the Appendix for some analysis of this). This "averaging", while avoiding aliasing (or spectral distortion) will reduce the frequency content of the heart rate signal and preclude the signal from representing fast transitions in heart rate. The main conclusion, however, is that a 5 -s sampling period is, in general, adequate for heart rate recording at all exercise intensities. This is not only a useful result for practitioners recording heart rate, during exercise and non-exercise (low-intensity) regimens, but has important implications for then 


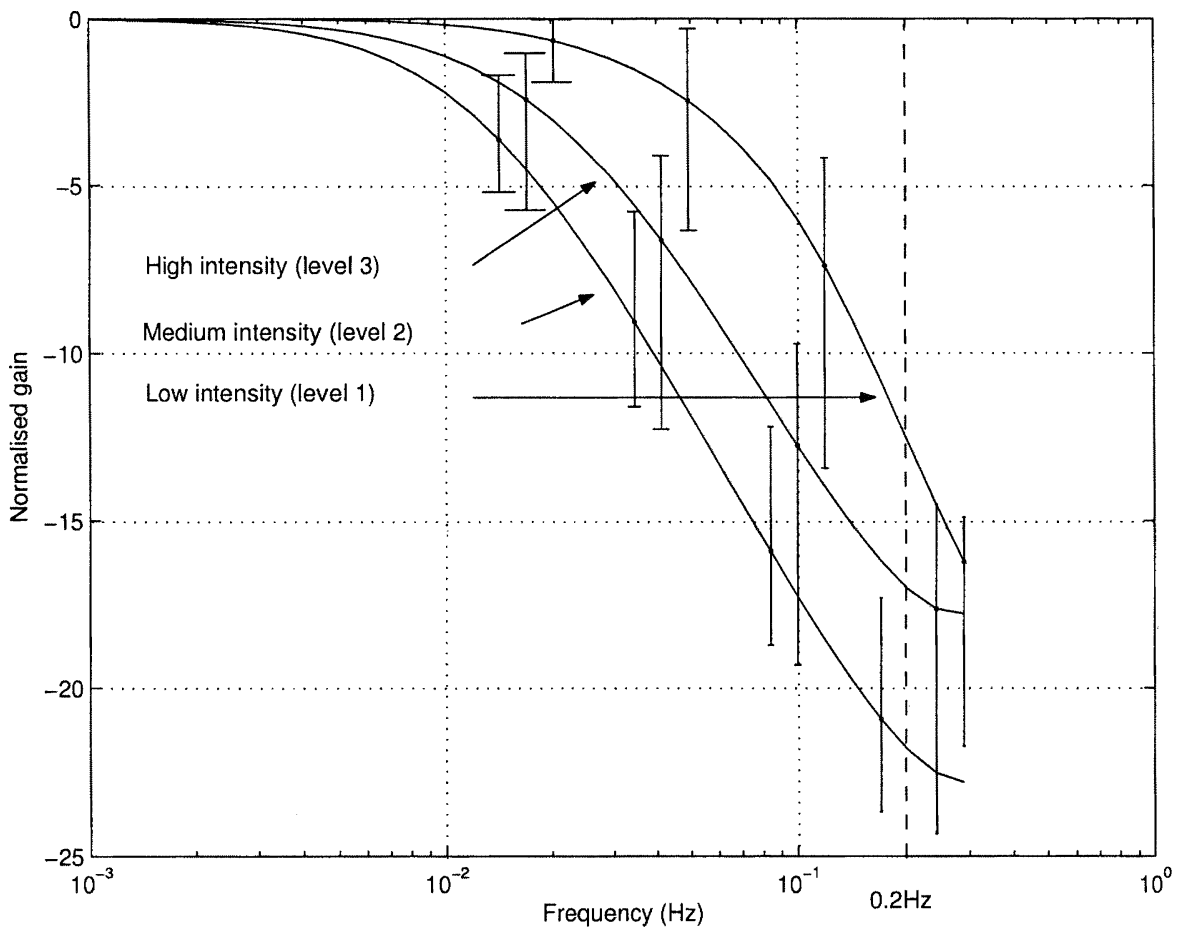

Figure 8. Mean frequency responses for each exercise intensity.

development of methods for non-invasive measurement of anaerobic threshold (Ringwood, 1999).

Given that the Nyquist-Shannon criterion is an upper bound on the sampling period, the results presented demonstrate that, if desired, a longer sampling period (than $5 \mathrm{~s}$ ) could be used with high and medium exercise intensities. However, the extra gain (20 s as opposed to $5 \mathrm{~s}$ ) is not sufficient to warrant the extra complexity of a variable sampling period and most exercise regimens will need to pass through the low-intensity phase in any case.

It should also be noted that the analysis in this paper was carried out on (interpolated) data sampled at $1 \mathrm{~Hz}$. This is perfectly reasonable, since the authentically reproduced frequency range $(0-$ $0.5 \mathrm{~Hz}$ ) more than covers the frequency range of interest $(0-0.1 \mathrm{~Hz})$.

\section{References}

Athens, M., Dertouzos, M. L., \& Mason, S. J. (1974). Systems, networks and computation: Multivariate methods. New York: McGraw-Hill.

Bakker, H. K., Struikenkamp, R. S., \& De Vries, G. A. (1980). Dynamics of ventilation, heart rate and gas exchange: Sinusoidal and impulse work loads in man. fournal of Applied Physiology, 51, 289-301.

Capurro, A., Diambra, L., \& Malta, C. P. (2003). Model for the heart beat-to-beat time series during meditation. Physica A: Statistical Mechanics and Its Applications, 327(1/2), 168-173.

Carroll, T., Godsen, R., \& Stone, S. (1991). How well does the Polar Vantage XL Heart Rate Monitor estimate actual heart rate? Medicine and Science in Sports and Exercise, 23 (suppl. 4), S14.

De Boor, C. (1978). A practical guide to splines. New York: Springer.
Dempsey, E. J., \& Westwick, D. T. (2004). Identification of Hammerstein models with cubic spline nonlinearities. IEEE Transactions on Biomedical Engineering, 51, 237-245.

Guild, S.-J., Austin, P. C., Navakatikyan, M., Ringwood, J. V., \& Malpas, S. (2001). Dynamic relationship between sympathetic nerve activity and renal blood flow: A frequency domain approach. American fournal of Physiology: Regulatory and Integrative Physiology, 281, R206-R212.

Kawada, T., Yanagiya, Y., Uemura, K., Miyamoto, T., Zheng, C., Li, M. et al. (2003). Input-size dependence of the baroreflex neural arc transfer characteristics. American fournal of Physiology: Heart and Circulation Physiology, 284, H404-H415.

Laukkanen, R., \& Virtanen, P. (1998). Heart rate monitors-state of the art. Fournal of Sports Sciences, 16, S3-S7.

Ljung, L. (1999). System identification: Theory for the user (2nd edn.). Englewood Cliffs, NJ: Prentice-Hall.

Ljung, L., \& Glad, T. (1994). Modelling of dynamic systems. Englewood Cliffs, NJ: Prentice-Hall.

MacFarlane, D. J., Fogarty, B. A., \& Hopkins, W. G. (1989). The accuracy and variability of commercially available heart rate monitors. New Zealand fournal of Sports Medicine, 17(4), 51 - 53.

McCarthy, M. (2005). Non-invasive determination of anaerobic threshold. MEngSc thesis, Department of Electronic Engineering, NUI Maynooth.

Mitra, S. K., \& Kaiser, J. F. (Eds.) (1993). Handbook for digital signal processing. New York: Wiley.

Murphy, J. (1999). Anaerobic threshold measurement in cyclists. MEng dissertation, School of Electronic Engineering, Dublin City University.

Nyquist, H. (1928). Certain topics in telegraph transmission theory. IEE Transactions, 47, 617-644.

Polar Electro Inc. (1997). PCBA receiver technical specificationsNRM receiver module. Product \#380142. Woodbury, NY: OEM Division, Polar Electro Inc.

Polar Electro Inc. (2003). http://www.polar.fi (accessed 13 July 2003).

Ringwood, J. V. (1999). Anaerobic threshold measurement using dynamic neural network models. Computers in Biology and Medicine, 29, 259-271. 
Ruha, A., Sallinen, S., \& Nissila, S. (1997). A real-time microprocessor QRS detector system with a 1-ms timing accuracy for the measurement of ambulatory HRV. IEEE Transactions on Biomedical Engineering, 44, 159-167.

Shannon, C. E. (1949). Communications in the presence of noise. Proceedings of the IREE, 37, 10-21.

Takagi, T., \& Sugeno, M. (1985). Fuzzy identification of systems and its application to modelling and control. IEEE Transactions on Systems, Man and Cybernetics, 15, 116-132.

Tulppo, M. P., Makikallio, T. H., Takala, T., Seppanen, T., \& Huikuri, H. (1996). Quantitative beat-to-beat analysis of heart rate dynamics during exercise. American fournal of Physiology: Heart and Circulation Physiology, 271, H244-H252.

Vaidyanathan, P. P. (2001). Generalisations of the sampling theorem: Seven decades after Nyquist. IEEE Transactions on Circuits and Systems, 48, 1094-1109.

Wigertz, O. (1970). Dynamics of ventilation and heart rate in response to sinusoidal work load in man. Fournal of Applied Physiology, 29, 208-218.

Yamamoto, Y., Kawakami, Y., Nakamura, Y., Mokushi, K., Mutoh, Y., \& Miyashita, M. (1988). A new method for detecting anaerobic threshold from heart rate recording. IEEE Engineering in Medicine and Biology Society 10th Annual International Conference, 1, 265-266.

\section{Appendix}

An averaging operation, where $n$ samples of a (heart rate) signal are averaged over a 5-s period, may be expressed as a Finite Impulse Response (FIR) filter of the form:

$v_{k}=\frac{1}{n}\left(x_{k}+x_{k-1}+\cdots+x_{k-n+1}\right)=\frac{1}{n} \sum_{i=k-n+1}^{k} x_{i}$ where: $v$ is the filter output, in the time domain; $x$ is the filter input, in the time domain; and $\Delta T$, the sampling period, is $\Delta T=5 / n$, representing the interval between heart beats.

Following averaging, the heart rate may be resamplcd on a 5 -s interval using:

$$
V_{k \Delta T_{5}}=V_{n k \Delta T}=v_{n k}
$$

where $\Delta T_{5}$ represents the 5 -s interval. Figure 9 shows the frequency response of averaging filters, as described in equation (18), for values of $n$ of 5 , 10 , and 15, corresponding to heart rates of 60 , 120 , and 180 beats $\cdot \min ^{-1}$ respectively. Since the sampling period (between heart beats) varies with number of beats in a 5 -s period (i.e. $n \Delta T=5$ ), the magnitude responses are almost perfectly consistent, with the minor variation being due to the change in filter order, $n$. If desired, the magnitude of the sidelobes can be reduced with appropriate windowing (Mitra \& Kaiser, 1993), but since no information is available on the proprietary processing schemes employed by commercial heart rate monitor manufacturers, it is beyond the scope of this paper to speculate as to which might be employed. The sidelobes in Figure 8 result from the rectangular window employed implicitly in (18), where the resulting magnitude response is of the form $|\sin (f) / f|$.

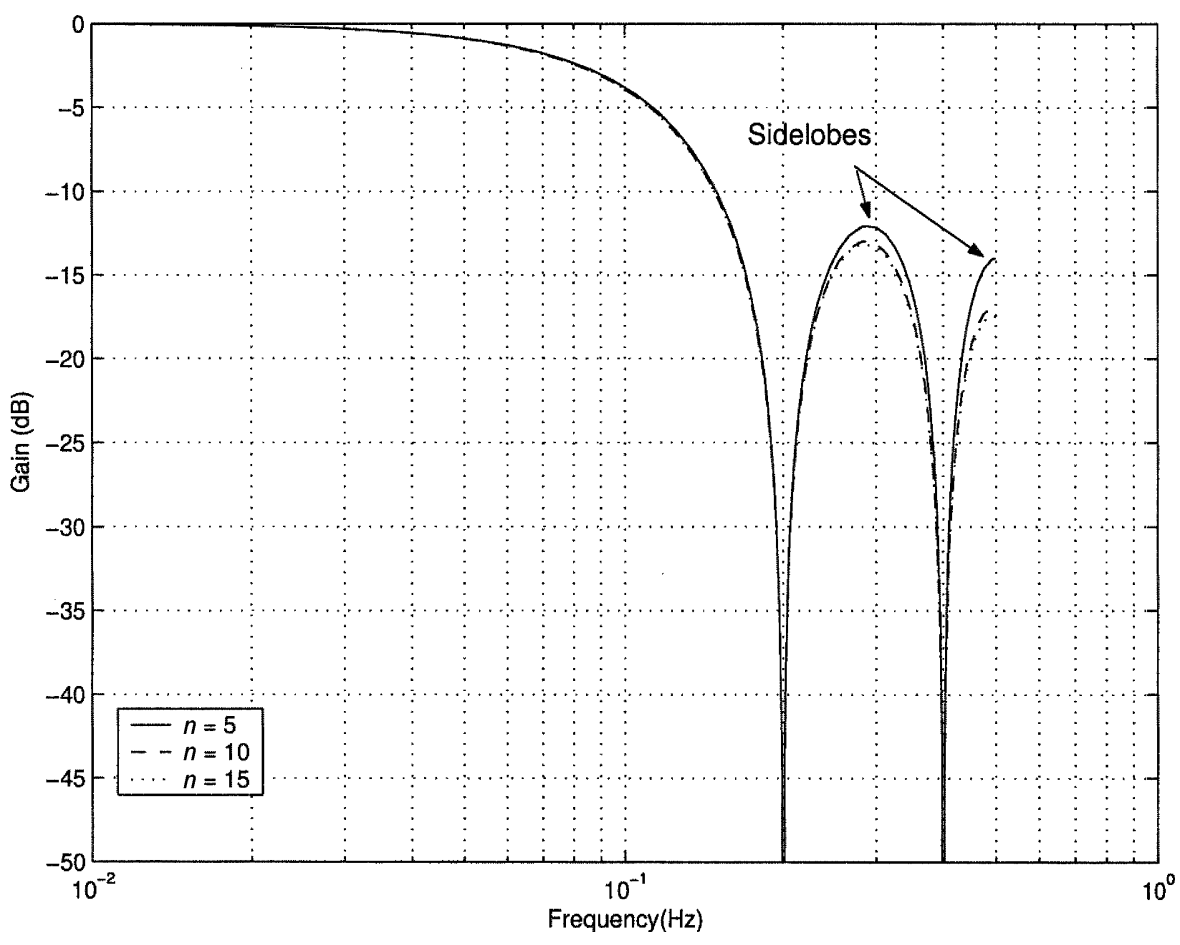

Figure 9. Frequency response of averaging filters. 